\title{
A Simple Method for Choosing the Parameters of a Two Degree-of-Freedom Tuned Vibration Absorber
}

\section{Authors}

S.-J. Jang ${ }^{1 *}$, M. J. Brennan ${ }^{2}$, E. Rustighi ${ }^{3}$, and H.-J. Jung ${ }^{4}$

\section{Affiliations}

${ }^{1}$ : Centre for Urban Energy System Research, Korea Institute of Science and Technology, 39-1 Hawolgok-dong, Seongbuk-gu, Seoul, 136-791, Republic of Korea

2: Departamento de Engenharia Mecânica, UNESP, SP 15385-000, Ilha Solteira, Brazil

3: Institute of Sound and Vibration Research, University of Southampton, Hampshire, SO17 1BJ, UK

${ }^{4}$ : Department of Civil and Environmental Engineering, Korea Advanced Institute of Science and Technology, 373-1 Guseong-dong, Yuseong-gu, Daejeon, 305-701, Republic of Korea

*Corresponding author

E-mail: seonjun.jang@gmail.com

Tel: 82(0)2 9585677

Fax: 82(0)2 9585689 


\begin{abstract}
In this paper a new method for choosing the parameters of a Two Degree-of-Freedom (2DOF) Tuned Vibration Absorber (TVA) with translational and rotational degrees of freedom is described. The dynamic stiffness approach is used to model the device, which is constrained to move in the translational direction and to rotate. The choice of the parameters involves a procedure similar to that proposed by Den Hartog in the optimization of a single DOF TVA, in which the invariant (fixed) points of the frequency response function of the TVA and the host structure are used to determine the stiffness of the TVA for a given mass. In this paper the approach is extended and applied to the design of a 2DOF TVA, and a numerical procedure is used to determine the optimum amount of damping. The method is simple and easy to implement. A numerical example is presented to compare the performance of the 2DOF TVA designed using the method described here with the optimal 2DOF TVA. It is shown that the performances of the TVAs are similar, validating the new approach.
\end{abstract}

\title{
Keyword
}

two degree-of-freedom tuned vibration absorber, dynamic stiffness approach, fixed point theory 


\section{Introduction}

A conventional Tuned Vibration Absorber (TVA) is a Single Degree-of-Freedom (SDOF) system, which can be used to suppress a troublesome resonance or to attenuate the vibration of a structure at a particular forcing frequency. The SDOF TVA is a well established vibration control device which has attracted the interest of many researchers since it was firstly proposed by Frahm about a century ago [1] in the configuration of a mass-spring system. Ormondroyd and Den Hartog [2] showed that the introduction of damping in the TVA can broaden the frequency range over which the device is effective. Den Hartog [3] suggested a two-step design technique which is still in use today. First, the resonance frequency is chosen, and second the optimal level of damping is determined. This technique is based on the observation of the existence of two invariant (fixed) points in the frequency response function of the TVA and the host structure, which do not change with the damping in the TVA. The TVA is optimally tuned when the responses of the system at the two fixed points is set to be equal [3]. The optimal value of damping is then obtained by averaging the two damping coefficients obtained by setting to zero the derivative at the two fixed points $[3,4]$. This pragmatic approach is a simple design procedure which gives very good performance. However, this approach does not give an optimal design for the system response to broadband vibration. Crandall and Mark [5] proposed an optimization method for the optimal design of the TVA which minimizes the $H_{2}$ norm of the response of the system. When the host structure is damped, different optimal values are obtained and different design techniques have been proposed [6,7] to determine the optimal parameters of the TVA. More recently, Asami et al. [8] summarized the above research when the host structure is damped for three different optimization criteria: $H_{\infty}$ optimization, $H_{2}$ optimization and most rapid 
attenuation of free vibration. In recent times, the simple configuration of an SDOF TVA has been modified with the aim of improving the performance of such a device. For example, Snowdon [9] considered a three-element TVA in which the damper is connected in series with a spring and showed that such a device can be more effective than a conventional TVA of the same mass. More recently Cheung and Wong [10-12] studied a TVA where the damper is connected to the ground instead of the mass of the host structure. Other researchers have tried also to introduce nonlinearities in the TVA to improve the performance, for example $[13,14]$.

The use of multiple TVAs has also been considered. Snowdon [9] suggested the use of an undamped TVA of small mass tuned to the problematic frequency connected in parallel with a conventional damped TVA. He showed an improvement in the response of the conventional TVA by the introduction of a significant trough in the frequency response function. The initial idea of using two TVAs in parallel has been further developed providing different design guidelines by Snowdon et al [15]. Brennan [16] demonstrated that the use of multiple TVAs in parallel tuned to slightly different frequencies can be used to create an effective broadband device. Techniques for the optimal design of multiple TVAs have been recently proposed by Zuo and Nayfeh [17]. Zuo [18] has also proposed to connect multiple TVAs in series. He showed that this configuration is more effective than an SDOF TVA of the same mass, and he provided ready-to-use design charts for the use of two TVAs in series.

In this paper a single TVA with two degrees-of-freedom (2DOF) is of interest. Zuo and Nayfeh [19] first introduced the concept of a multi-degree-of-freedom TVA of this type. They suggested a numerical procedure to find the optimal stiffness and damping coefficients of the TVA that maximize the minimal damping of the multiple modes in a prescribed frequency range. They treated the springs and dampers as position and velocity feedback elements 
respectively and numerically solved for the static decentralized control problem. Jang and Choi [20] considered an undamped multi-degree-of-freedom TVA, and tuned its six modes to those of the main structure, providing analytical tuning conditions for its design. Zuo and Nayfeh also presented a more detailed study on a 2DOF TVA [21] obtained by connecting a planar mass to an SDOF main structure by two connection points. They obtained the optimal values of the two sets of spring and damper coefficients which minimize the $\mathrm{H}_{2}$ or $\mathrm{H}_{\infty}$ norms of the transmissibility when the system is subject to base excitation. They showed that the 2DOF TVA can outperform two separate SDOF TVAs of the same mass. Recently Jang et al [22] provided a further insight in the physics of the 2DOF TVA by examining the behavior of a 2DOF TVA with very small moment of inertia. In this case the model of the device can be simplified and reasons why it marginally outperforms the conventional SDOF TVA [21] were given.

In this paper, the authors develop a model of a $2 \mathrm{DOF}$ with translational and rotational degrees of freedom using the dynamic stiffness approach. Preliminary results of this study were given in [23]. First, the dynamic stiffness of the TVA is derived, and this is used to study the basic dynamics of the TVA as the moment of inertia is increased. Next, a design process to determine the parameters of the 2DOF TVA based on Den Hartog's method of fixed points is described. Lewis [24] proved the existence of fixed points in the frequency response of undamped multi-degree-of-freedom host structure with a damped SDOF TVA attached, and Ozer and Royston [25] extended Den Hartog's method to such systems. Here, Den Hartog's method of fixed points is extended to the case of a 2DOF TVA attached to an SDOF host structure. Although this does not give an optimal result, it gives a result that is very close to 
the optimal result, in the same way as when the method is applied to a SDOF TVA. It does, however, have the advantage of being simple to apply.

\section{DYNAMIC STIFFNESS MODEL OF THE TVA}

The 2DOF TVA of interest attached to an undamped SDOF host structure is shown in Fig. 1. The TVA mass $m$ with moment of inertia $J$ is connected to the host structure of mass $m_{s}$ and stiffness $k_{s}$ through two sets of springs and dampers $k_{1}, c_{1}$ and $k_{2}, c_{2}$, respectively. It may translate in the vertical direction and rotate around the mass center, as the host structure, which is subject to base excitation, is constrained to vibrate in the vertical direction only. The distances from the mass center to the set of springs and dampers are given by $d_{1}$ and $d_{2}$. The 2DOF TVA is shown in Fig 2(a). It may be described by a mass $m$ and complex, frequency dependent dynamic stiffness $K$ as shown in Fig. 2(b).

To determine the dynamic stiffness $K$, the mass is blocked to translation, but rotation is allowed, i.e., a pinned boundary condition is applied at the mass centre. Such dynamic stiffness will take into account the rotational inertia of the mass. The effect of the translational mass will be taken into account later. Using the mechanical impedance approach [26], the two forces that pass through the set of springs and dampers are given by

$$
\begin{aligned}
& F_{1}=\alpha X_{1}+\beta X_{2} \\
& F_{2}=\beta X_{1}+\gamma X_{2}
\end{aligned}
$$


where $\quad \alpha=\left.\frac{F_{1}}{X_{1}}\right|_{X_{2}=0}, \quad \gamma=\left.\frac{F_{2}}{X_{2}}\right|_{X_{1}=0}$, and $\beta=\left.\frac{F_{2}}{X_{1}}\right|_{X_{2}=0}=\left.\frac{F_{1}}{X_{2}}\right|_{X_{1}=0}$. Noting that the dynamic stiffness of the two sets of springs and dampers is given by [26] $K_{1}=\mathrm{j} \omega c_{1}+k_{1}$, $K_{2}=\mathrm{j} \omega c_{2}+k_{2}$ respectively, the forces and the moments about mass center of the mass element can be summed to give

$$
\alpha=\frac{1}{\frac{1}{K_{1}}+\frac{1}{K_{2}\left(\frac{d_{2}}{d_{1}}\right)^{2}-\frac{J \omega^{2}}{d_{1}^{2}}}}, \quad \gamma=\frac{1}{\frac{1}{K_{2}}+\frac{1}{K_{1}\left(\frac{d_{1}}{d_{2}}\right)^{2}-\frac{J \omega^{2}}{d_{2}^{2}}}}, \quad \beta=\frac{1}{\frac{1}{K_{1}\left(\frac{d_{1}}{d_{2}}\right)}+\frac{1}{K_{2}\left(\frac{d_{2}}{d_{1}}\right)}-\frac{1}{\frac{K_{1} K_{2} d_{1} d_{2}}{J \omega^{2}}}} .
$$

The specific conditions for the system in Fig. 2 are $F=F_{1}+F_{2}$ and $X=X_{1}=X_{2}$, so Eqs. $(1 \mathrm{a}, \mathrm{b})$ combine to give

$$
\frac{F}{X}=(\alpha+2 \beta+\gamma)
$$

Substituting the values obtained for $\alpha, \beta$ and $\gamma$ into Eq. (2) results in the following expression for the dynamic stiffness

$$
K=\frac{F}{X}=\frac{\left(d_{1}+d_{2}\right)^{2} K_{1} K_{2}-J \omega^{2}\left(K_{1}+K_{2}\right)}{d_{1}^{2} K_{1}+d_{2}^{2} K_{2}-J \omega^{2}} .
$$

When the frequency and/or the moment of inertia is large such that $J \omega^{2}$ is large, then the mass is prevented from rotation, and Eq. (3) reduces to

$$
K=\left(K_{1}+K_{2}\right)
$$

which is simply the dynamic stiffness of the parallel combination of stiffness and damping elements of an SDOF TVA. When the frequency and/or the moment of inertia is small such that $J \omega^{2}$ is small, then the force transfers from one spring damper set to the other through the mass, largely unattenuated so that Eq. (3) reduces to 


$$
K=\frac{\left(d_{1}+d_{2}\right)^{2} K_{1} K_{2}}{d_{1}^{2} K_{1}+d_{2}^{2} K_{2}}
$$

which represents a series combination of dynamic stiffness $q_{2} K_{1}$ and $q_{1} K_{2}$ where $q_{i}=\left(\frac{d_{1}+d_{2}}{d_{i}}\right)^{2}$. In both of these extreme cases, the inertia of the mass element plays no part in the dynamics of the absorber as discussed in [22], but in the general case, away from these extremes, the inertia does have an effect, causing a peak in the dynamic stiffness $K$ at a frequency of $\omega=\sqrt{\frac{d_{1}^{2} K_{1}+d_{2}^{2} K_{2}}{J}}$ and a trough at a frequency of $\omega=\sqrt{\frac{\left(d_{1}+d_{2}\right)^{2} K_{1} K_{2}}{\left(K_{1}+K_{2}\right) J}}$. It is this dynamic effect on the effectiveness of the TVA that is of interest in this paper. Note that $q_{1}$ and $q_{2}$ determine the contribution to the system of $K_{1}$ and $K_{2}$ respectively. When $d_{1} / d_{2} \rightarrow 0, q_{1} / q_{2} \rightarrow \infty$ and $K \approx K_{2}$; and when $d_{1} / d_{2} \rightarrow \infty, q_{1} / q_{2} \rightarrow 0$ and $K \approx K_{1}$. When $d_{1}=d_{2}, q_{1}=q_{2}=1 / 4$ and the two sets of dynamic stiffness elements contribute equally to the overall stiffness; additionally, if both sets of stiffness and damping are the same, then the total dynamic stiffness is simply twice that of one of the sets of elements.

The dynamic stiffness the 2DOF TVA is the series combination of $K$ and the mass $m$ as shown in Fig. 2(b), and is given by

$$
K_{\mathrm{TVA}}=\frac{1}{\frac{1}{K}-\frac{1}{\omega^{2} m}} .
$$

Substituting for $K$ from Eq. (3) into Eq. (6) results in 


$$
K_{\mathrm{TVA}}=\frac{-\omega^{2} m\left(K_{1} K_{2}-b \omega^{2}\left(K_{1}+K_{2}\right)\right)}{b m \omega^{4}-\left(\left(b+q_{1} m\right) K_{1}+\left(b+q_{2} m\right) K_{2}\right) \omega^{2}+K_{1} K_{2}}
$$

where $b=\frac{J}{\left(d_{1}+d_{2}\right)^{2}}$ is the scaled inertia term.

\section{Design of a 2DOF TVA}

\subsection{Undamped 2DOF TVA}

To investigate the behavior of the 2DOF TVA, it is assumed that the springs and dampers are placed equidistant from the centre of mass, such that $d_{1}=d_{2}$, so $q_{1}=q_{2}=1 / 4$. This does not affect the generality of the approach, but reduces the algebraic complexity in this illustration of the methodology. In this case, Eq. (7) reduces to

$$
K_{\mathrm{TVA}}=\frac{-4 \omega^{2} m\left(K_{1} K_{2}-b \omega^{2}\left(K_{1}+K_{2}\right)\right)}{4 b m \omega^{4}-(4 b+m)\left(K_{1}+K_{2}\right) \omega^{2}+4 K_{1} K_{2}} .
$$

The peaks in the dynamic stiffness of the 2DOF TVA effectively become the anti-resonances of the complete system when the TVA is attached to the host structure. Therefore, they play a crucial role in the dynamics of the overall system. To determine the frequencies $\omega_{1,2}$ at which they occur, the damping coefficients $c_{1}$ and $c_{2}$ are set to zero, and the denominator in Eq. (8) is set to zero and solved to give

$$
\hat{\omega}_{1,2}=\frac{\left(1+\mu_{k}\right)\left(1+\mu_{m}\right) \pm \sqrt{\left(1+\mu_{k}\right)^{2}\left(1+\mu_{m}\right)^{2}-16 \mu_{k} \mu_{m}}}{4 \mu_{m}}
$$

where 


$$
\hat{\omega}_{1,2}=\frac{\omega_{1,2}^{2}}{2 k_{1} / m}, \mu_{k}=\frac{k_{2}}{k_{1}} \text {, and } \mu_{m}=\frac{4 b}{m} \text {. }
$$

Fixing the ratio between the inertia term $4 b$ and the mass $m$ of the device, the gap between the two tuning frequencies $\omega_{1,2}$ only depends on the stiffness ratio $\mu_{k}$. It is thus possible to tune the two anti-resonance frequencies of the system by choosing the parameters $b, m, k_{1}$ and $k_{2}$. Figure 3 shows these frequencies as a function of $\mu_{m}$ for various values of the stiffness ratio $\mu_{k}$. The advantage of a 2DOF TVA compared to an SDOF TVA is that a smaller mass can be used to obtain the same performance, because of the exploitation of the rotational inertia in the 2DOF TVA.

\subsection{Damped 2DOF TVA}

In this section, the damped 2DOF TVA is used to attenuate the single resonant peak of an undamped SDOF system subject to base excitation. The aim is to reduce the vibration of the host structure over a frequency band and not at a particular harmonic excitation frequency. To achieve this, the fixed point technique for the damped SDOF TVA [2] is extended to the 2DOF TVA. This approach makes the design process very quick and simple. The performance of the TVA designed using strategies based on optimal control theory [21] is compared with the proposed method.

Since the fixed points occur if the system has a single damper, a damped 2DOF TVA with single damper $c_{1}$ is now considered. Moreover, as previously shown, the presence of the second damper only influences the frequency response at high frequencies, well above the tuned frequencies of the TVA. Its effect on the performance around the resonance frequencies 
of interest is thus negligible. It is noted that the optimal results obtained from numerical work in [21] were also for a 2DOF TVA with a single damper, i.e., $c_{2}=0$.

For an undamped SDOF host structure, with dynamic stiffness $K_{s}=k_{s}-\omega^{2} m_{s}$, the motion transmissibility from the base to the mass of the host structure is given by [22]

$$
T=\frac{X_{s}}{X_{e}}=\frac{k_{s}}{K_{s}+K_{\mathrm{TVA}}}
$$

The magnitude of $T$ can be obtained by substituting for $K_{s}$ and $K_{\mathrm{TVA}}$. This can be rewritten in terms of non-dimensional variables to give

$$
|T|=\left[\frac{\left(-\Omega^{4}+2 v^{2} \Omega^{2}-4 v^{4} \mu_{a} \gamma_{1}\left(1-\mu_{a}\right)\left(2-\gamma_{1}\right)\right)^{2}+4 \zeta^{2}\left(\Omega^{3}-4 v^{3} \mu_{a}\left(1-\mu_{a}\right)\left(2-\gamma_{1}\right) \Omega\right)^{2}}{\left(\begin{array}{l}
\Omega^{6}-\left(1+2 v^{2}+2 v^{2} \mu_{a} \mu\right) \Omega^{4} \\
+\left(2 v^{2}+4 v^{4} \mu_{a} \gamma_{1}\left(1-\mu_{a}\right)(1+\mu)\left(2-\gamma_{1}\right)\right) \Omega^{2} \\
-4 v^{4} \mu_{a} \gamma_{1}\left(1-\mu_{a}\right)\left(2-\gamma_{1}\right)
\end{array}\right)^{2}+4 \zeta^{2}\left(\begin{array}{l}
-v\left(1+\mu_{a} \mu\right) \Omega^{5}+v\left(1+4 v^{2} \mu_{a}\left(1-\mu_{a}\right)(1+\mu)\left(2-\gamma_{1}\right)\right) \Omega^{3} \\
-4 v^{3} \mu_{a}\left(1-\mu_{a}\right)\left(2-\gamma_{1}\right) \Omega
\end{array}\right)^{2}}\right]^{\frac{1}{2}}
$$

where $\Omega=\frac{\omega}{\omega_{s}}, \omega_{s}=\sqrt{\frac{k_{s}}{m_{s}}}, m_{a}=\frac{4 b m}{4 b+m}=m\left(1+\mu_{m}^{-1}\right)^{-1}, \quad k_{a}=\frac{k_{1}+k_{2}}{2}, \omega_{a}=\sqrt{\frac{k_{a}}{m_{a}}}, \quad v=\frac{\omega_{a}}{\omega_{s}}$, $\mu=\frac{m}{m_{s}}, \quad \mu_{a}=\frac{m_{a}}{m}=\left(1+\mu_{m}^{-1}\right)^{-1}, \quad \zeta=\frac{c_{1}}{2 m_{a} \omega_{a}}$, and $\gamma_{1}=\frac{k_{1}}{k_{a}}=2\left(1+\mu_{k}\right)^{-1}$.

Ormondroyd and Den Hartog [2] discovered the existence of two fixed points in the frequency response of a force excited undamped SDOF system with an attached an SDOF damped TVA. They observed that all the curves pass through the same two points regardless of the damping in the TVA. Moreover these two points move depending on the ratio of the stiffness of the TVA to the host structure. They then found the most suitable parameters for the TVA by 
1. Adjusting the stiffness of the TVA so that the response at the invariant points was the same

2. Adjusting the damping so that the frequency response curves had a horizontal tangent through one of them (to reduce the frequency response to a minimum).

The existence of invariant points can also be observed when a 2DOF TVA is mounted on a SDOF undamped system, such as that shown in Fig 1. Figure 4 shows the frequency response given by Eq. (11) for the parameters used in [21]. When the damping is zero there are two anti-resonances, where the response is zero, as discussed in the last section, and there are three resonances. When the damping is infinite the TVA behaves as an SDOF TVA, since the mass can only pivot around the point of connection with $K_{1}$ and thus only one antiresonance and two resonances are observed. However, when moderate damping is present the amplitudes at the resonance frequencies are reduced and the response at the anti-resonances is no longer zero. When the damping ratio is $\zeta=0.1482$, an optimal design, as discussed in [21] is obtained, and this is illustrated next.

The four frequency response functions in Fig. 4 intersect at four invariant points (expressed as red dots). These are the fixed points [2] and are used to determine the optimum parameters of the 2DOF TVA. The location of the fixed points can be found observing that at certain frequencies Eq. (11) is not dependent on damping [3]. Thus, equating the transmissibilities at $\zeta=0$ and $\infty$ from Eq. (11) yields [27]

$$
\frac{A}{B}= \pm \frac{C}{D}
$$




\section{where}

$$
\begin{aligned}
& A=-\Omega^{4}+2 v^{2} \Omega^{2}-4 v^{4} \mu_{a} \gamma_{1}\left(1-\mu_{a}\right)\left(2-\gamma_{1}\right) \\
& B=\Omega^{6}-\left(1+2 v^{2}+2 v^{2} \mu_{a} \mu\right) \Omega^{4}+\left(2 v^{2}+4 v^{4} \mu_{a} \gamma_{1}\left(1-\mu_{a}\right)\left(2-\gamma_{1}\right)(1+\mu)\right) \Omega^{2}-4 v^{4} \mu_{a} \gamma_{1}\left(1-\mu_{a}\right)\left(2-\gamma_{1}\right) \\
& C=\nu \Omega^{3}-4 v^{3} \mu_{a}\left(1-\mu_{a}\right)\left(2-\gamma_{1}\right) \Omega \\
& D=-v\left(1+\mu_{a} \mu\right) \Omega^{5}+v\left(1+4 v^{2} \mu_{a}\left(1-\mu_{a}\right)(1+\mu)\left(2-\gamma_{1}\right)\right) \Omega^{3}-4 v^{3} \mu_{a}\left(1-\mu_{a}\right)\left(2-\gamma_{1}\right) \Omega
\end{aligned}
$$

The transmissibilites with zero and infinite damping ratio are in opposite phase at the fixed points. The negative sign on the right side of Eq. (12) is thus taken and expanding it yields

$$
\begin{aligned}
(2+ & \left.\mu_{a} \mu\right) \Omega^{8}-2\left(1+2 v^{2}+2 v^{2} \mu_{a}\left(\mu+\left(2-\gamma_{1}\right)\left(1-\mu_{a}\right)(2+\mu)\right)\right) \Omega^{6} \\
& +4 v^{2}\left(1+\mu_{a}\left(2-\gamma_{1}\right)\left(1-\mu_{a}\right)\left(2+4 v^{2}+v^{2}\left(2 \gamma_{1}+\mu\left(2+\gamma_{1}\right)\left(1+\mu_{a}\right)\right)\right)\right) \Omega^{4} \\
& -8 v^{4} \mu_{a}\left(2-\gamma_{1}\right)\left(1-\mu_{a}\right)\left(2+\gamma_{1}\left(1+4 v^{2} \mu_{a}\left(2-\gamma_{1}\right)\left(1-\mu_{a}\right)\left(1+\mu_{a}\right)\right)\right) \Omega^{2} \\
& +36 v^{6} \gamma_{1} \mu_{a}^{2}\left(2-\gamma_{1}\right)^{2}\left(1-\mu_{a}\right)^{2}=0
\end{aligned}
$$

Solving Eq. (13) yields four $\Omega_{\text {fixed }, i}^{2}(i=1 \cdots 4)$ which are related to the frequencies of the fixed points. The sum of them is another invariant that can be used in the TVA design and is given by

$$
\sum_{i}^{4} \Omega_{\text {fixed }, i}^{2}=\frac{2\left(1+2 v^{2} \mu_{a}\left(\mu+\left(2-\gamma_{1}\right)\left(1-\mu_{a}\right)(2+\mu)\right)\right)}{2+\mu_{a} \mu} .
$$

It is also possible to find the frequencies, $\Omega_{\text {const }, i}^{2}(i=1 \cdots 4)$ corresponding to the four points with the same amplitude of response $|T|_{\text {const }}$ from Eq. (11) with $\zeta \rightarrow \infty$, which results in

$$
|T|_{\text {const }}=\left|\frac{\Omega^{2}-4 v^{2} \mu_{a}\left(1-\mu_{a}\right)\left(2-\gamma_{1}\right)}{-\left(1+\mu_{a} \mu\right) \Omega^{4}+\left(1+4 v^{2} \mu_{a}\left(1-\mu_{a}\right)(1+\mu)\left(2-\gamma_{1}\right)\right) \Omega^{2}-4 v^{2}\left(1-\mu_{a}\right)\left(2-\gamma_{1}\right)}\right| .
$$

Squaring both sides of Eq. (15) and rearranging yields 


$$
\begin{aligned}
& |T|_{\text {const }}^{2}\left(1+\mu_{a} \mu\right)^{2} \Omega^{8}-2|T|_{\text {const }}^{2}\left(1+\mu_{a} \mu\right)\left(1+4 v^{2} \mu_{a}(1+\mu)\left(2-\gamma_{1}\right)\left(1-\mu_{a}\right)\right) \Omega^{6} \\
& +\left(\begin{array}{l}
-1+|T|_{\text {const }}^{2}+8|T|_{\text {const }}^{2} v^{2} \mu_{a}\left(2-\gamma_{1}\right)\left(1-\mu_{a}\right) \\
\left(2+\mu+\mu_{a}\left(4 v^{2}+\mu-2 v^{2}\left(\gamma_{1}\left(1-\mu_{a}\right)(1-\mu)^{2}+2 \mu_{a}(1+\mu)^{2}-2 \mu(2+\mu)\right)\right)\right)
\end{array}\right) \Omega^{4} . \\
& -8 v^{2} \mu_{a}\left(2-\gamma_{1}\right)\left(1-\mu_{a}\right)\left(-1+|T|_{\text {const }}^{2}+4|T|_{\text {const }}^{2} v^{2} \mu_{a}(1+\mu)\left(1-\mu_{a}\right)\left(2-\gamma_{1}\right)\right) \Omega^{2} \\
& -16 v^{4} \mu_{a}^{2}\left(2-\gamma_{1}\right)^{2}\left(1-\mu_{a}\right)^{2}\left(1-|T|_{\text {const }}^{2}\right)=0
\end{aligned}
$$

From Eq. (16), the sum of the squares of the four frequencies, $\Omega_{\text {const, } i}^{2}$ is given as

$$
\sum_{i}^{4} \Omega_{\mathrm{const}, i}^{2}=\frac{2\left(1+4 v^{2} \mu_{a}\left(1-\mu_{a}\right)\left(1+\mu_{a}\right)\left(2-\gamma_{1}\right)\right)}{1+\mu_{a} \mu}
$$

The first step in Den Hartog's method to determine the optimum parameters for an SDOF TVA is to find the values of stiffness and mass which align the two fixed points. In the 2DOF TVA the parameters ( $\mu, \mu_{a}, \gamma_{1}$, and $v$ ) need to be adjusted to align the four fixed points in the same amplitude of response. To ensure that the response does not have any peaks in the frequency range of interest, such that the response is relatively flat, Eqs. (14) and (17) are set to be equal. This results in

$$
v=\sqrt{\frac{1}{2+2 \mu_{a}\left(-4(1+\mu)+\gamma_{1}\left(1-\mu_{a}\right)\left(2+\mu\left(3+\mu_{a} \mu\right)\right)+\mu_{a}\left(4-\mu\left(6+\mu\left(1-2 \mu_{a}\right)\right)\right)\right)}} .
$$

By fixing the mass ratio $\mu$, it is possible to find the value of $v$ for any pair of $\left(\mu_{a}, \gamma_{1}\right)$ in the ranges $0 \leq \mu_{a} \leq 1$ and $0 \leq \gamma_{1} \leq 2$ (these ranges correspond to the minimum and maximum values of the non-dimensional parameters). Substituting the values into Eq. (13), the frequencies at which the fixed points occur can be obtained. However, Eq. (18) gives only a necessary, but not sufficient condition for the four fixed points to have the same transmissibility. Thus another constraint is needed. As a measure of the difference between the transmissibilities of the fixed points, the standard deviation $s\left(\mu, \mu_{a}, \gamma_{1}\right)$ is calculated. As 
with the SDOF case, this quantity does not vary with $\zeta$. Fig. 5 shows the standard deviation in the transmissibilities of the fixed points when the mass ratio $\mu$ has been fixed to 0.05 . As shown in Fig. 6, for every $\mu$ there are two minima in the functions, that is there are only two optimal values of the pair $\left(\mu_{a}, \gamma_{1}\right)$. The minimum values have been found numerically and their values as function of the mass ratio $\mu$ are shown in Fig. 6(a) and 6(b). Fig. 6(c) shows that the standard deviation is generally very small for the optimum values and becomes increasingly smaller for a larger mass ratio.

The parameters of the 2DOF TVA can be selected by the following two steps.

1. Fix the mass ratio, $\mu$. Fig. 5 can then be used to select the pair of $\mu_{a}$ and $\gamma_{1}$ which gives the smallest standard deviation and consequently the parameter $v$ is found from Eq. (18).

2. The damping ratio $\zeta$ which minimizes the frequency response is chosen. This could be done by taking the average of the values which bring to zero the derivative of the transmissibility at the fixed points, similar to Den Hartog's method. However, here a numerical method which minimizes the area of the transmissibility is used, to achieve a better performance.

\section{Numerical example}

To demonstrate the design process, a host structure consisting of an undamped SDOF system is considered. Assuming a mass ratio $\mu=0.05$, the two points where the standard 
deviation becomes very small correspond to $\gamma_{1}=0.8648, \mu_{a}=0.3809$ and $\gamma_{1}=1.088$, $\mu_{a}=0.5964$. Choosing $\gamma_{1}=1.088$ and $\mu_{a}=0.5964, v$ is found to be 0.9220 .

The non-dimensional frequencies of the fixed points are $0.8174,0.8495,0.9412$, and 1.0901 and the magnitude of transmissibility at these frequencies is $14.7 \mathrm{~dB}$ to one decimal place. Finally, substituting all the parameters into Eq. (11) and searching numerically for the damping coefficient which gives the minimum area of the transmissibility yields $\zeta=0.1510$. The obtained parameters of the 2DOF TVA are listed in Tab 1. Figure 7 gives the frequency response of the transmissibility for three damping values, in which it is shown that the four fixed points have the same amplitude.

The transmissibility of the system optimised according to the method proposed above is compared with that obtained in [21] where an optimal control approach was used to determine the optimum parameters of the TVA. The $\|H\|_{2}$ value obtained from [21] is 2.0189 and the design method proposed here gives an $\|H\|_{2}$ value of 2.0480 , which is only about $1.5 \%$ larger than the optimal value. For every pair of $\gamma_{1}$ and $\mu_{a}$, the optimal $\|H\|_{2}$ value of transmissibility of the system is found by numerical optimization for each $v$ and $\zeta$, and is illustrated in Fig. 8. The design point, (1.088, 0.5964) determined using the method in this papers (expressed as a white dot) is located very close to the optimum point. Figure 9 shows the transmissibility of the system in Fig 1 when the 2DOF TVA is optimized using the method in [21] and also when the parameters of the 2DOF TVA are chosen according to the method described in this paper. The performance of an optimally tuned SDOF TVA [4], which has an $\|H\|_{2}$ value of 2.1241, for comparison. It can be seen that the 
2DOF TVA designed using the method proposed above outperforms the SDOF TVA and slightly underperforms the optimal 2DOF TVA.

\section{Conclusions}

This paper has described a method of choosing the parameters of a 2DOF TVA by developing the method of fixed points proposed by Den Hartog. A dynamic stiffness model of a damped 2DOF TVA with translational and rotational degrees of freedom was first derived. It was shown that the dynamic characteristics of such a system are strongly influenced by the rotational inertia of the TVA. In particular, for a small moment of inertia, or at low frequency, the effect of the rotational inertia is negligible and the two spring-damper elements behave as if they are connected in series. For a large moment of inertia, or at high frequencies, the system reduces to a conventional SDOF TVA. Between these two extremes the characteristics of the TVA change allowing the performance of the TVA to be marginally enhanced with respect to a conventional device.

Design guidelines for the use of 2DOF TVA with an SDOF undamped host structure have been given. The design procedure guides the choice of stiffness and mass, leaving only the damping to be determined numerically. It has been shown that the performance of 2DOF TVA with parameters chosen using the simple and pragmatic design procedure given in this paper is broadly comparable to that of an optimally designed 2DOF TVA. 


\section{Acknowledgement}

This work was supported by the Korea Research Foundation Grant funded by the Korean Government (MOEHRD), (KRF-2007-357-D00009), and by FAPESP.

\section{References}

[1] H. Frahm, Device for damped vibrations of bodies, U.S. Patent No. 989958, 1909.

[2] J. Ormondroyd, J. P. Den Hartog, The theory of the dynamic vibration absorber. Transactions of the ASME, Journal of Applied Mechanics 49 (1928) A9-A22.

[3] J. P. Den Hartog, Mechanical Vibrations, 4th ed., McGraw-Hill New York, 1956, Reprinted by Dover, New York, 1985.

[4]J. E. Brock, A note on the damped vibration absorber. Transaction of the ASME, Journal of Applied Mechanics 13 (1946) A-284.

[5] S. H. Crandall, W. D. Mark, Random Vibration in Mechanical Systems, Academic Press, 1963.

[6] G. B. Warburton, E. O. Ayorinde, Optimum absorber parameters for simple systems. Earthquake Engineering and Structural Dynamics 8 (1980) 197-217.

[7] T. Asami, T. Wakasono, K. Kameoka, M. Hasegawa, H. Sekiguchi, Optimum design of dynamic absorbers for a system subjected to random excitation. Japan Society of Mechanical Engineers JSME International Journal, Vol. 34, No. 2 (1991) 218-226.

[8] T. Asami, O. Nishihara, A. M. Baz, Analytical solutions to $H_{\infty}$ and $H_{2}$ optimization of dynamic vibration absorbers attached to damped linear systems. Transaction of the ASME, Journal of Vibration and Acoustics 124 (2002) 284-295. 
[9] J. C. Snowdon, Dynamic vibration absorber that have increased effectiveness. Transaction of the ASME, Journal of Engineering for Industry 96 (1974) 940-945.

[10] Y. L. Cheung, W. O. Wong, Design of a non-traditional dynamic vibration absorber (L). Journal of the Acoustical Society of America 126 (2009) 564-567.

[11] Y. L. Cheung, W. O. Wong, H-infinity optimization of a variant design of the dynamic vibration absorber - revisited and new results. Journal of Sound and Vibration 330 (2011) $3901-3912$

[12] Y. L. Cheung, W. O. Wong, $H_{2}$ optimization of a non-traditional dynamic vibration absorber for vibration control of structures under random force excitation. Journal of Sound and Vibration 330 (2011) 1039-1044.

[13] S. S. Oueini, A. H. Nayfeh, J. R. Pratt, A nonlinear vibration absorber for flexible structures. Nonlinear Dynamics, Vol. 15, No. 3 (1998) 259-282.

[14] G. S. Agnes, D. J. Inman, Performance of nonlinear vibration absorbers for multidegrees-of-freedom systems using nonlinear normal modes. Nonlinear Dynamics, Vol. 25, No. $1-3(2001) 275-292$.

[15] J. C. Snowdon, A. A. Wolfe, R. L. Kerlin, The cruciform dynamic vibration absorber. Journal of the Acoustical Society of America, Vol. 75, No. 6 (1984) 1792-1799.

[16] M. J. Brennan, Characteristics of a wideband vibration neutraliser. Noise Control Engineering Journal, Vol. 45, No. 5 (1997) 1-8.

[17] L. Zuo, S. A. Nayfeh, Optimization of the Individual Stiffness and Damping Parameters in Multiple-Tuned-Mass-Damper Systems. Transaction of the ASME, Journal of Vibration and Acoustics 127 (2005) 77-83. 
[18] L. Zuo, Effective and robust vibration control using series multiple tuned-mass dampers. Transaction of the ASME, Journal of Vibration and Acoustics 131 (2009) 031003.

[19] L. Zuo, S. A. Nayfeh, Minimax optimization of multi-degree-of-freedom tuned mass dampers. Journal of Sound and Vibration 272 (2004) 893-908.

[20] S. J. Jang, Y. J. Choi, Geometrical design method of multi-degree-of-freedom dynamic vibration absorbers. Journal of Sound and Vibration 303 (2007) 343-356.

[21] L. Zuo, S.A. Nayfeh, The two-degree-of-freedom tuned-mass damper for suppression of single-mode vibration under random and harmonic excitation. Transactions of the ASME, Journal of Vibration and Acoustics 128 (2006) 56-65.

[22] S.-J. Jang, M. J. Brennan, E. Rustighi, Comparing the performance of optimally tuned dynamic vibration absorbers with very large or very small moment of inertia. Transactions of the ASME, Journal of Vibration and Acoustics 132 (2010) 034501.

[23] S.-J. Jang, M. J. Brennan, E. Rustighi, H.-J. Jung, Theoretical investigation of 2DOF vibrating system and its application to dynamic vibration absorber. Journal of Computational Structural Engineering Institute of Korea, Vol. 22, No. 4, (2009) 371-377. (In Korean)

[24] F. M. Lewis, The extended theory of the viscous vibration damper. Transaction of the ASME, Journal of Applied Mechanics 22 (1955) 377-382.

[25] M. B. Ozer, T. J. Royston, Extending Den Hartog's vibration absorber technique to multi-degree-of-freedom systems. Transactions of the ASME, Journal of Vibration and Acoustic 127 (2005) 341-350.

[26] P. Gardonio, M. J. Brennan, Advanced Applications in Acoustics, Noise and Vibration, edited by Fahy, F.J. and Walker J.G., Chapter 9. Mobility and Impedance Methods. Spon Press, London and New York, 2004. 
[27] W. O. Wong, Y. L. Cheung, Optimal design of a damped dynamic vibration absorber for vibration control of structure excited by ground motion. Engineering Structures 30 (2008) 282-286.

\section{Figures}

Fig. 1. Base-excited SDOF system with 2DOF TVA attached.

Fig. 2. A 2DOF TVA. (a) actual TVA, (b) equivalent model in which $K$ is the dynamic stiffness of the combination of stiffness, damping and rotational inertia of the TVA.

Fig. 3. Anti-resonance frequencies $\hat{\omega}_{1,2}$ of the 2DOF TVA for different stiffness ratios: $\mu_{k}=0.01(*), 0.1(\circ), 1$ (solid line), $10(\triangleleft)$, and $100(\square)$.

Fig. 4. The transmissibility amplitude of the system in Fig. 1 for different values of the damping ratio: dotted red line $\zeta=0$, thin green solid line $\zeta=0.05$, thick black solid line $\zeta=0.1482$, dashed blue line $\zeta=\infty$. The fixed points are expressed as red dots. $(\mu=0.05$, $\left.\mu_{a}=0.3781, v=1.0828, \gamma_{1}=0.8528\right)$

Fig. 5. Contour map for standard deviations of the magnitudes of the fixed points in $\mathrm{dB}$ for a fixed mass ratio of $\mu=0.05$.

Fig. 6. (a) and (b): Optimal values of the two pairs $\left(\mu_{a}, \gamma_{1}\right)$ which give the minimum standard deviation in the four invariant points. (c) minimum standard deviation for the two optimal pairs. Pair 1: continuous line, Pair 2: dashed line.

Fig. 7. Transmissibility of the system in Fig 1 with the following non-dimensional parameters: $\mu=0.05, \mu_{a}=0.5964, v=0.9220, \gamma_{1}=1.088$, and three different values of damping in the TVA; $\zeta=0$ (dotted red line), $\zeta=0.1510$ (solid blue line: optimal), and 
$\zeta=\infty$ (dashed green line). The horizontal line shows the leveled magnitude of transmissibility of fixed points.

Fig. 8. Contour map for optimal $\|H\|_{2}$ values in $\mathrm{dB}$ for every $\gamma_{1}$ and $\mu_{a}$. The white dot shows the proposed design point using the method described in this paper.

Fig. 9. Comparison of the transmissibilities of the system in Fig 1 when the parameters of the 2DOF TVA are chosen in different ways: optimal SDOF TVA [4] (dotted green line), optimal $\|H\|_{2}$ 2DOF TVA [21] (dashed red line), 2DOF TVA with design parameters given in Table 1 (solid blue line)

\section{Tables}

Table 1. Design parameters for a damped 2DOF TVA applied to a main structure 


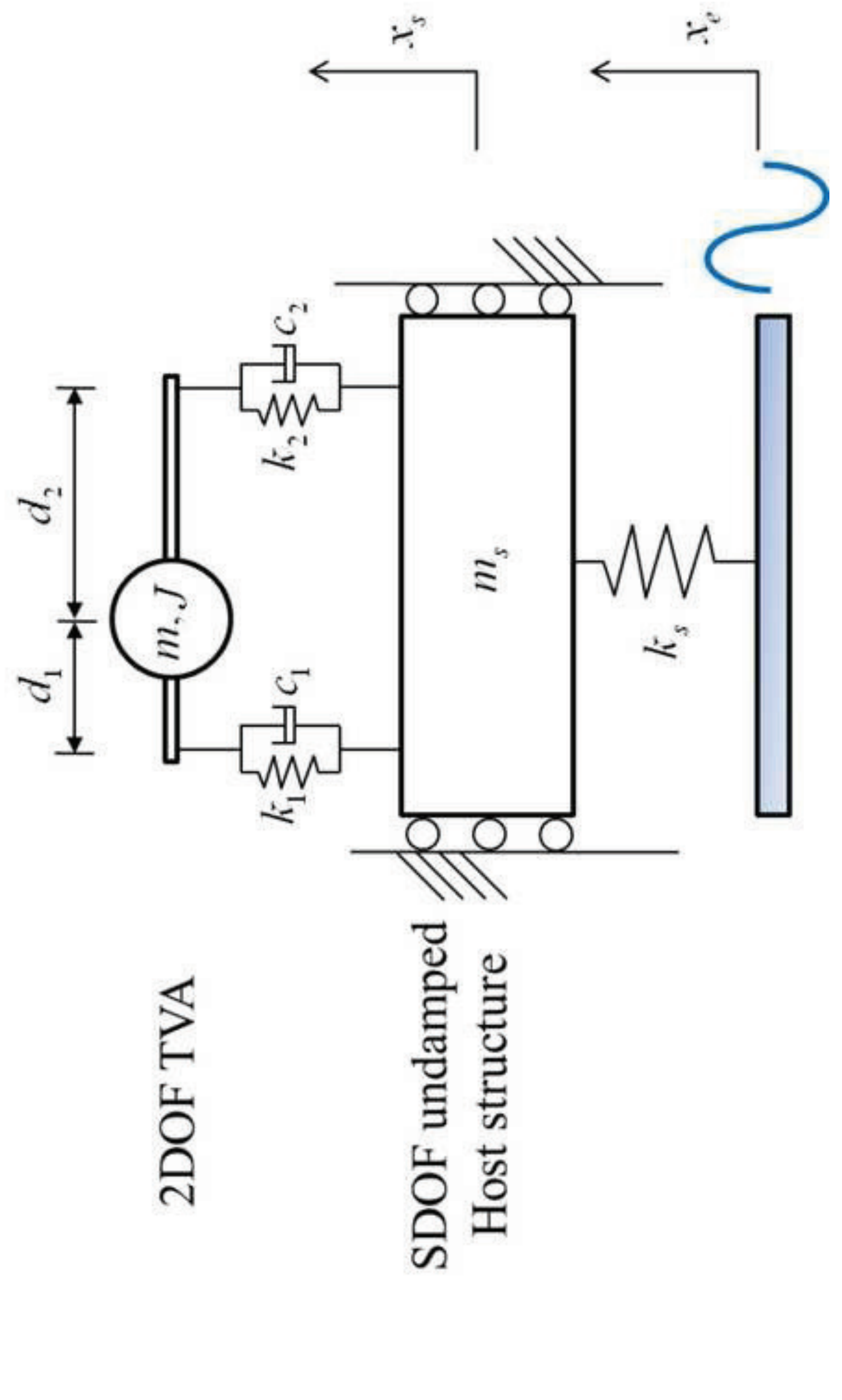




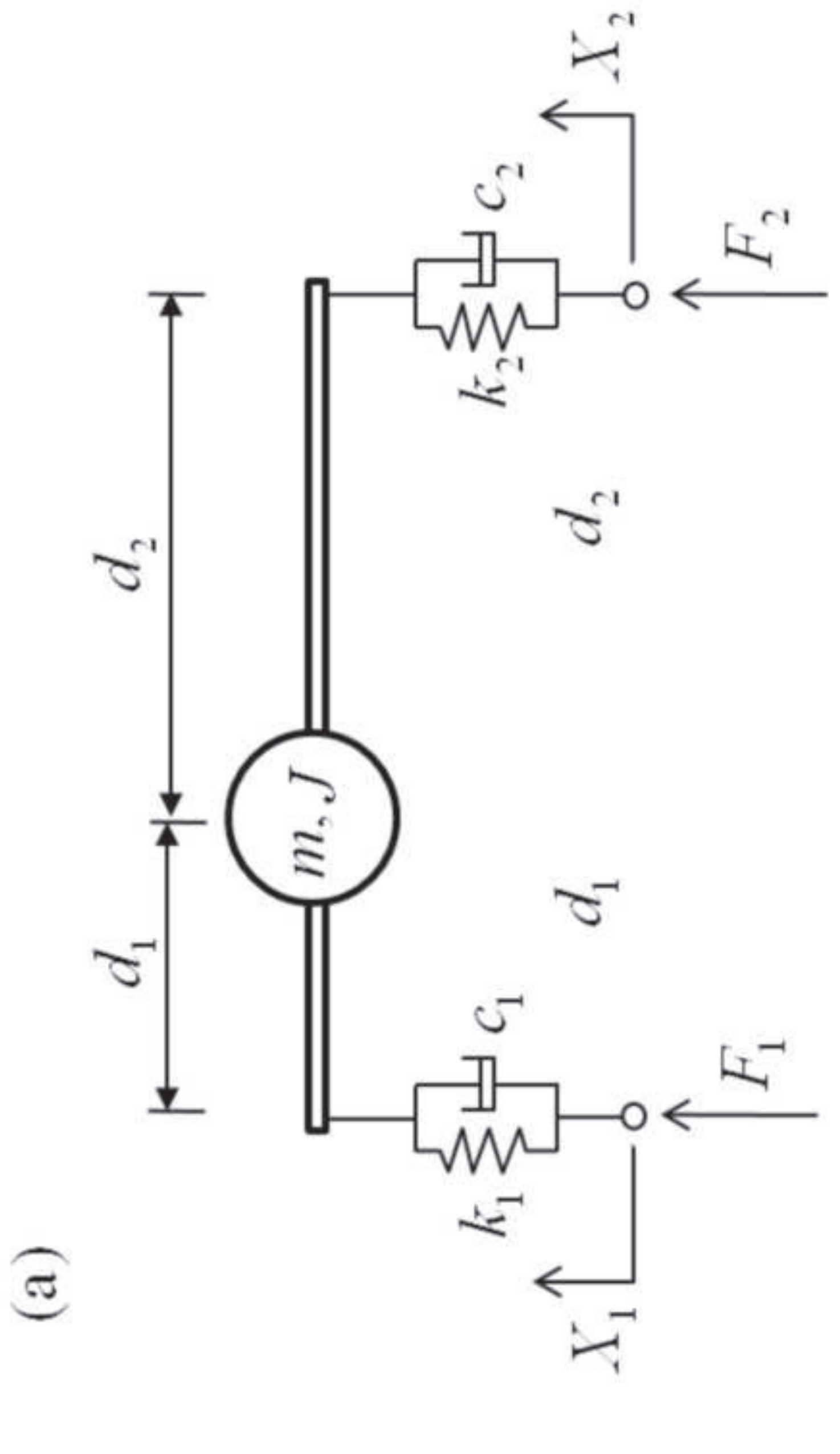


Figure 2b

Click here to download high resolution image<smiles>[194In]</smiles>

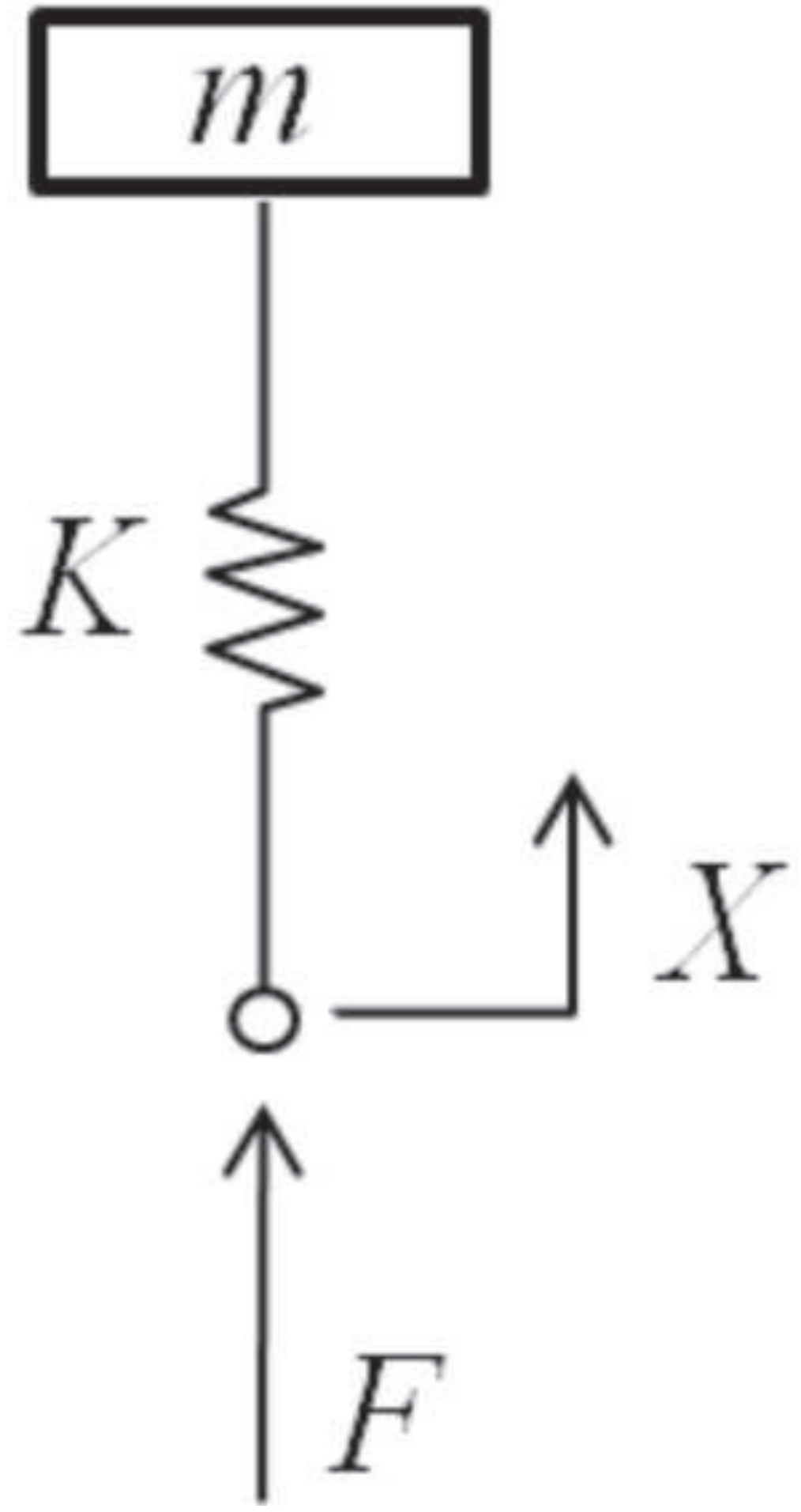




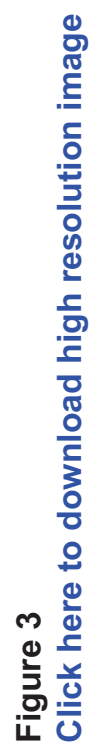

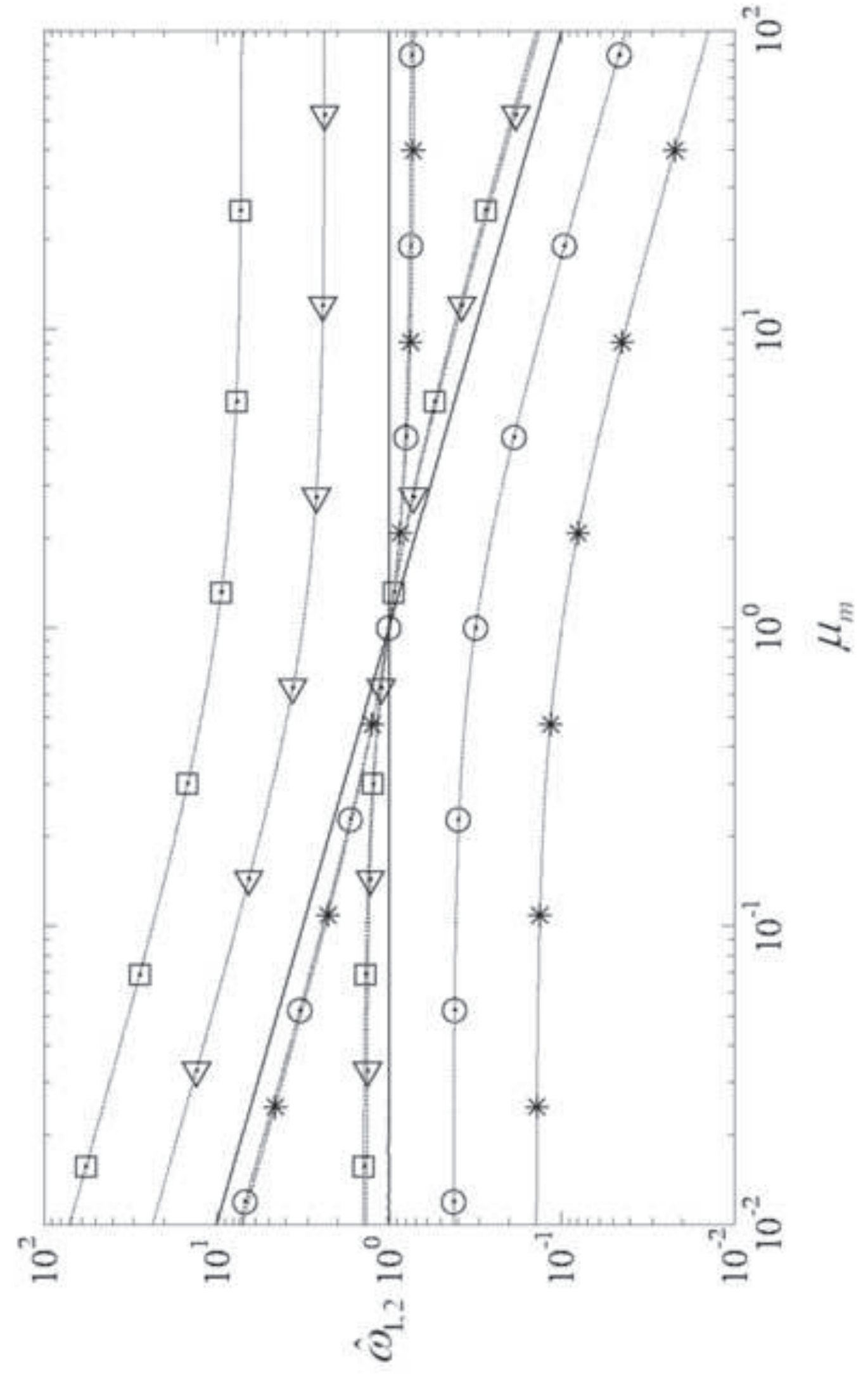




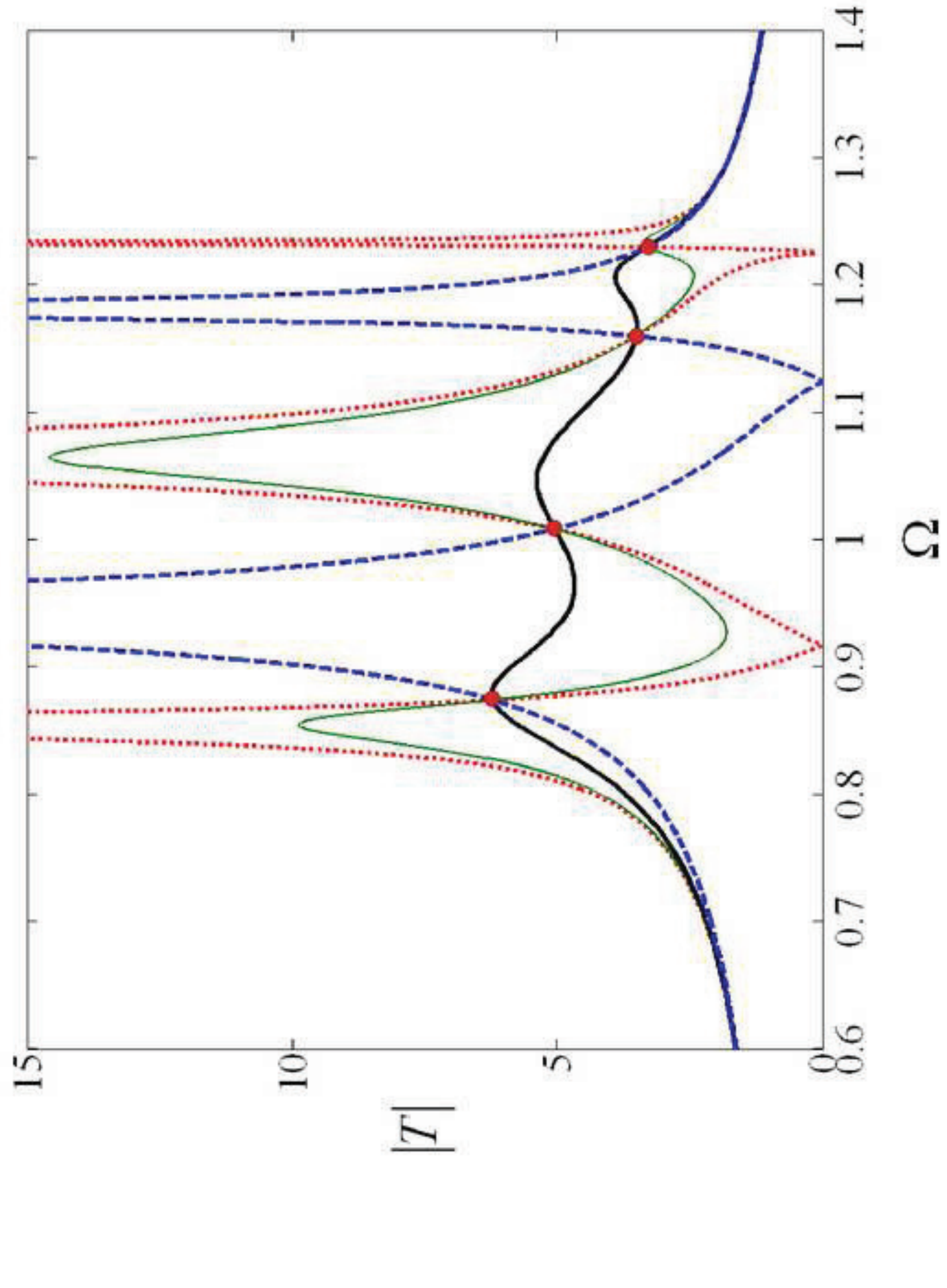



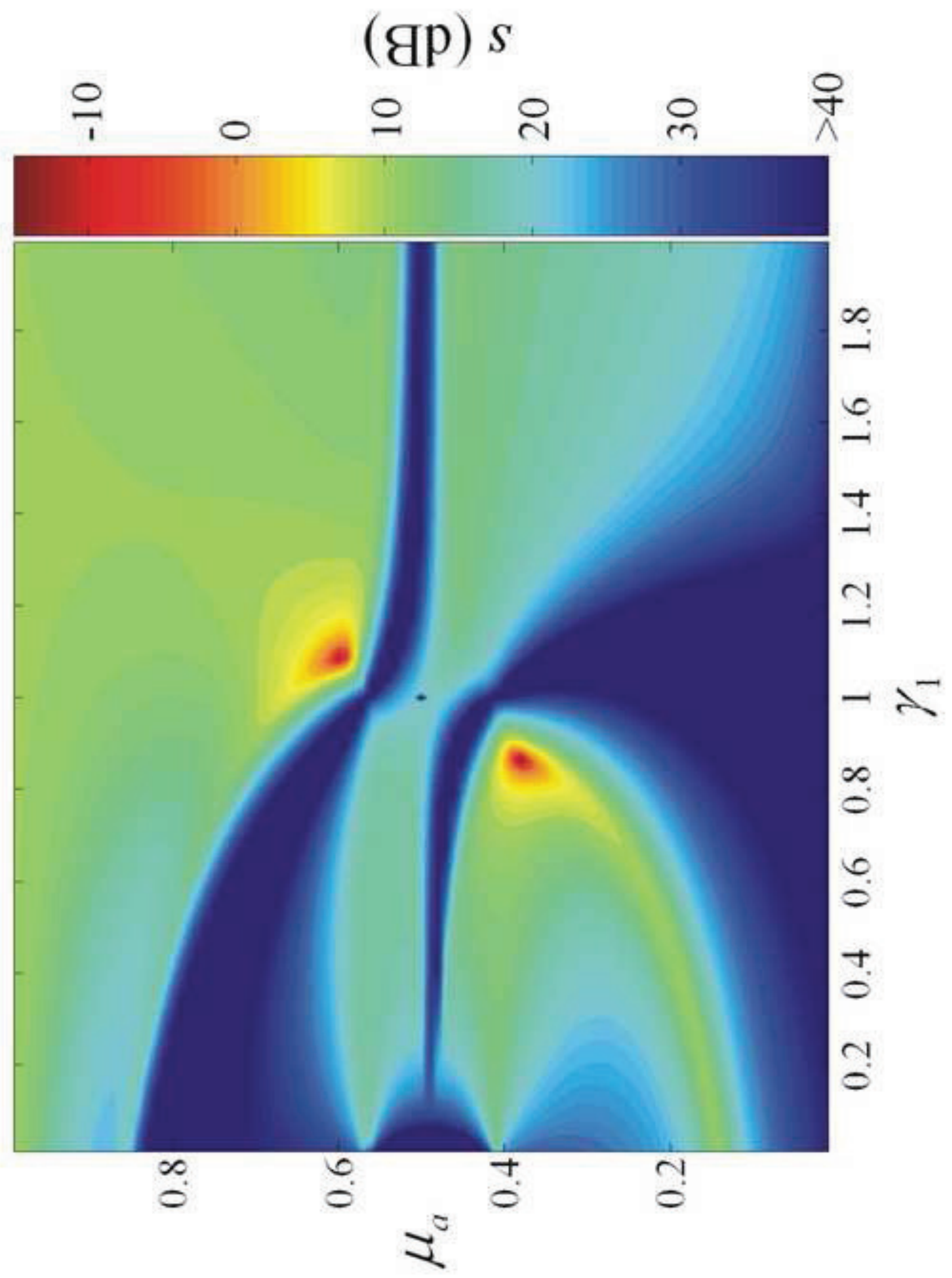

in

는

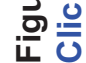




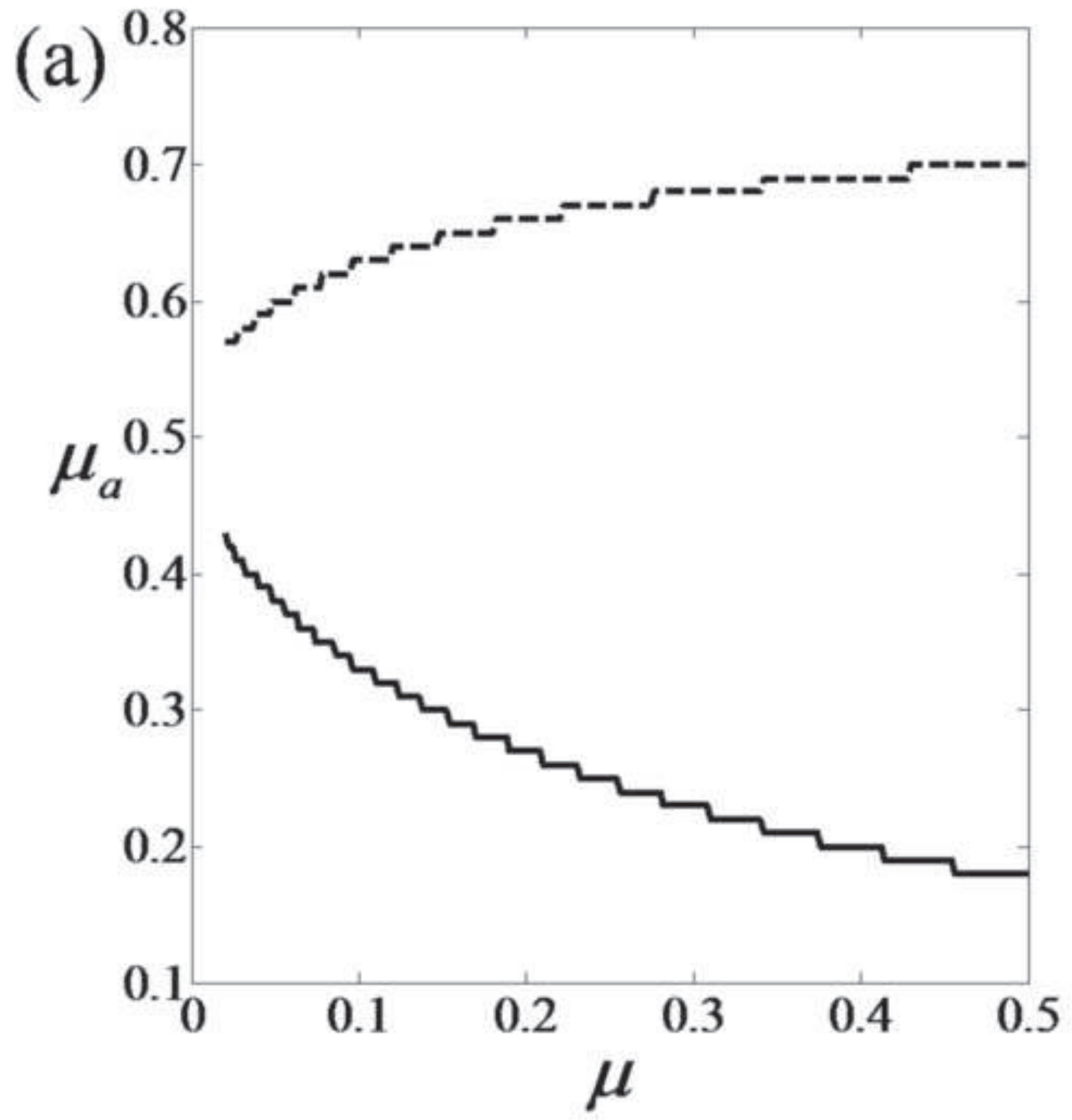



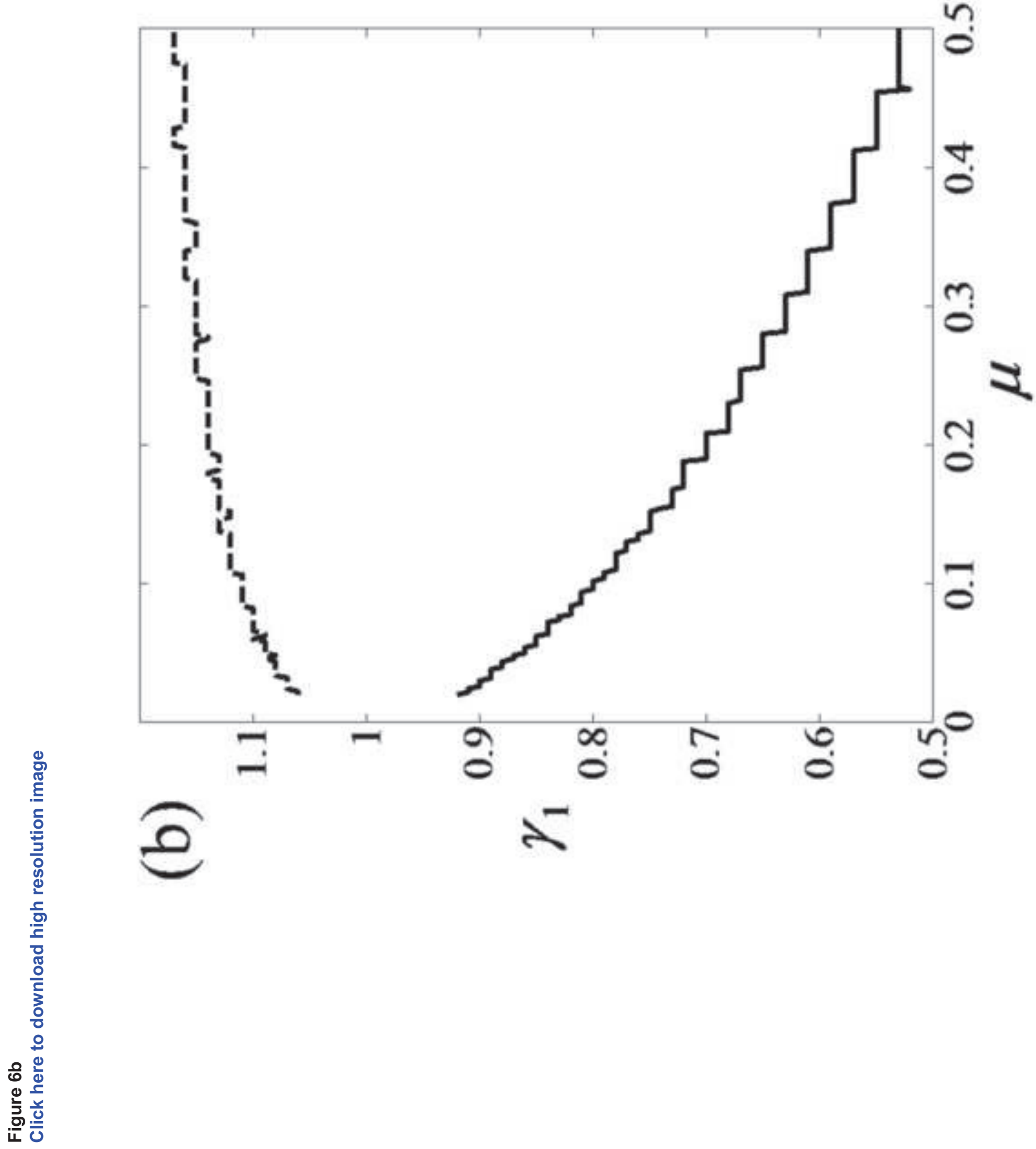


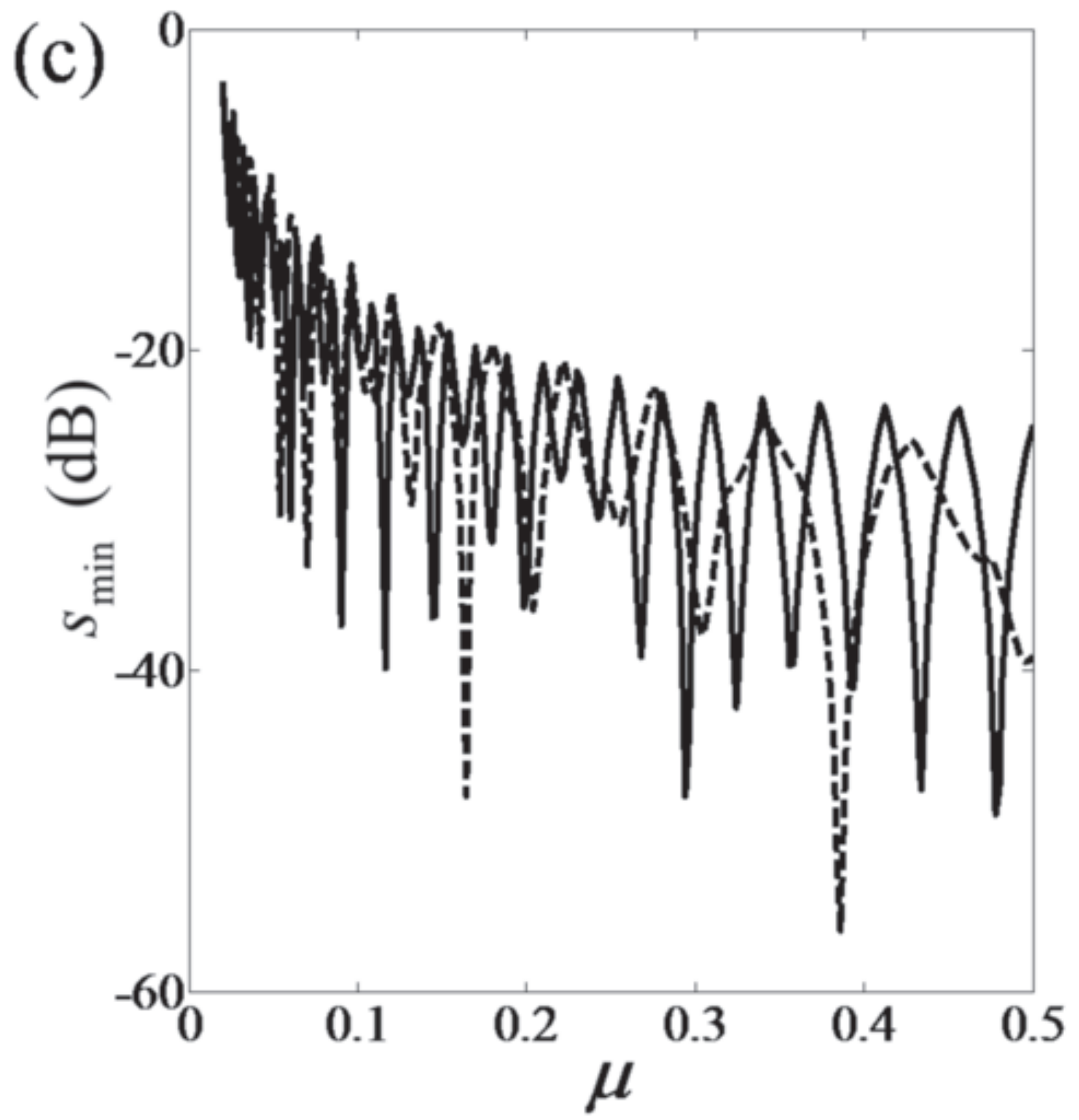




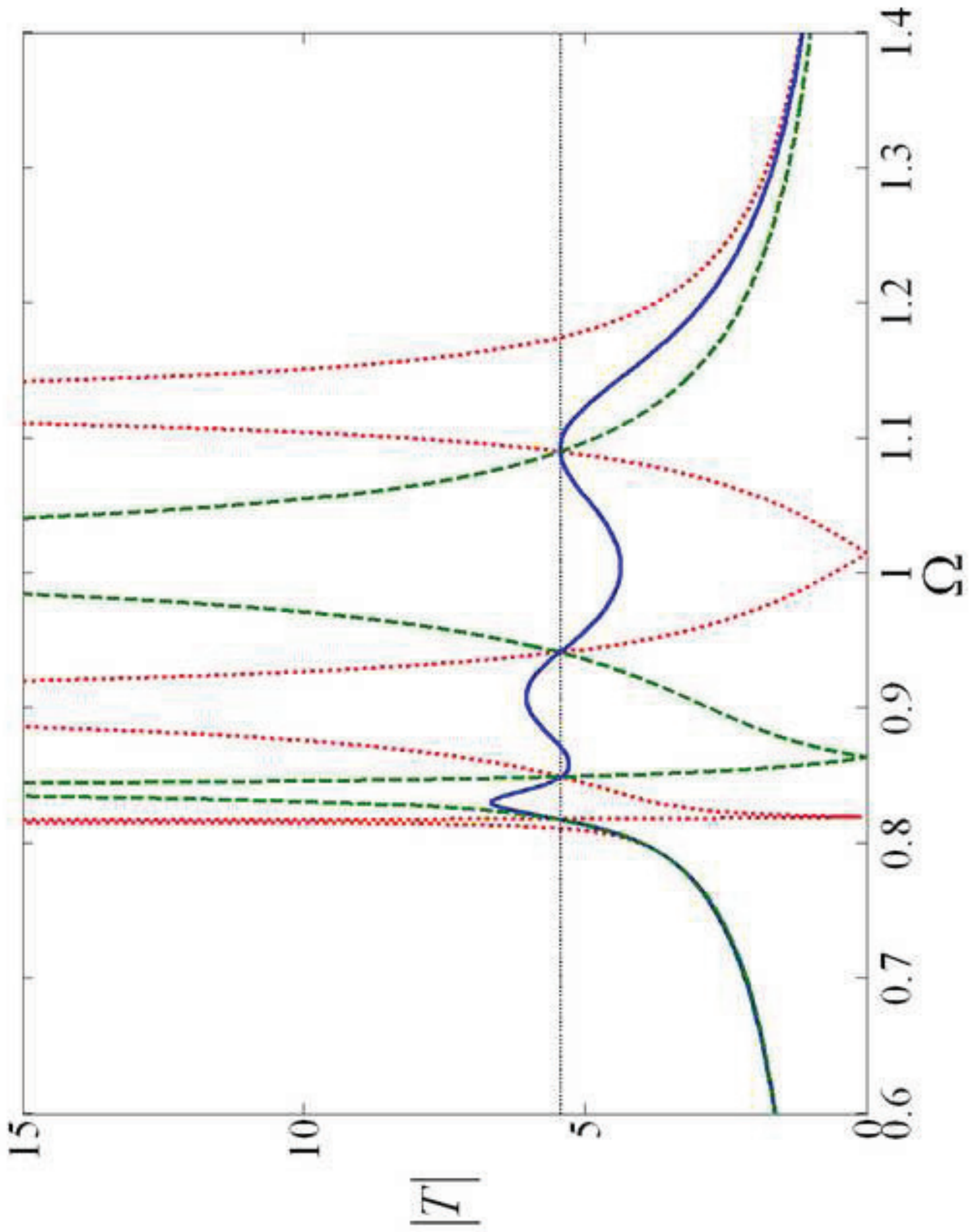




$$
\begin{aligned}
& \text { (gp) }{ }^{\tau}\|H\| \\
& \infty \simeq \simeq \pm \cong \simeq \text { त }
\end{aligned}
$$
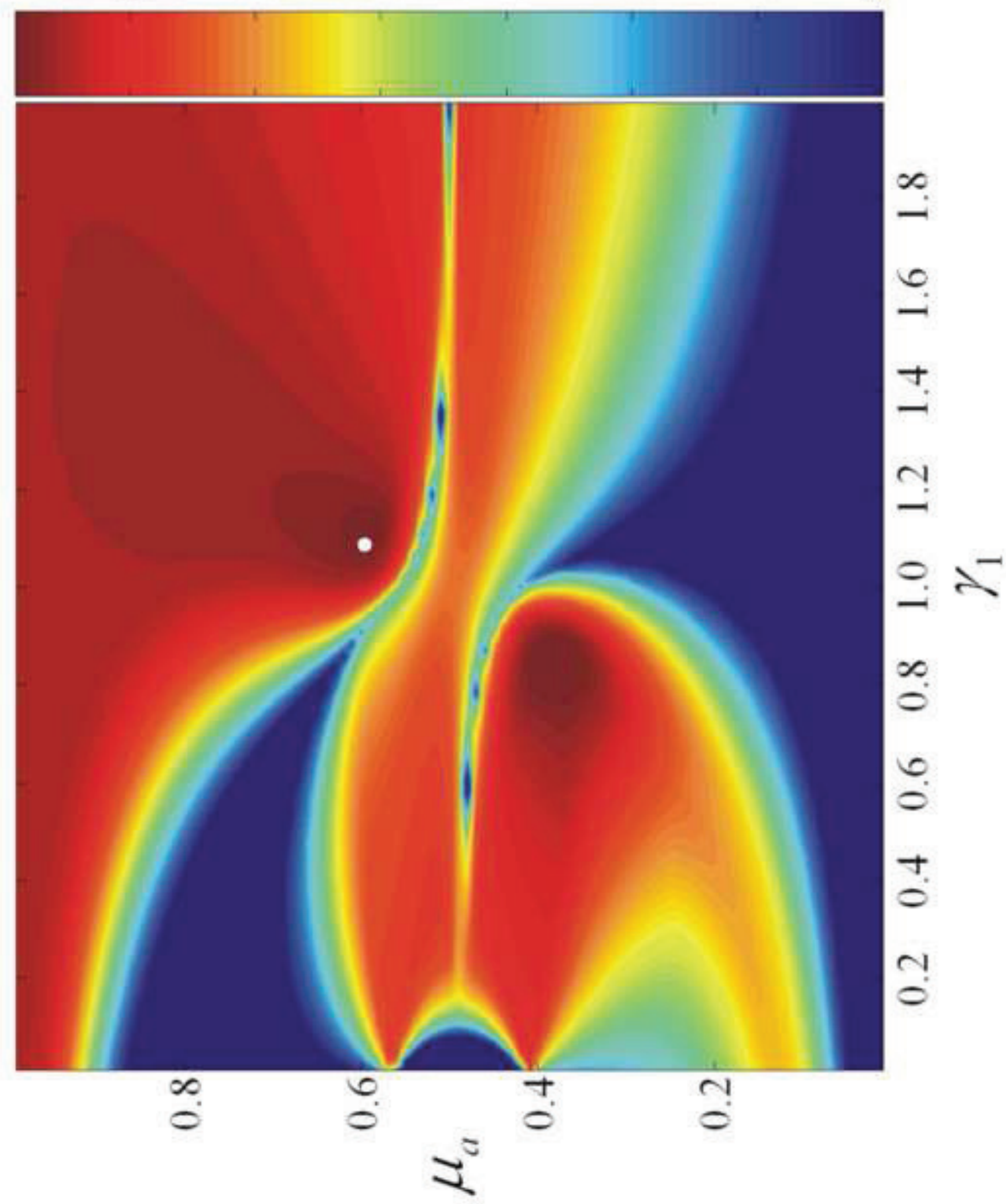

$\infty$

인

응

证 


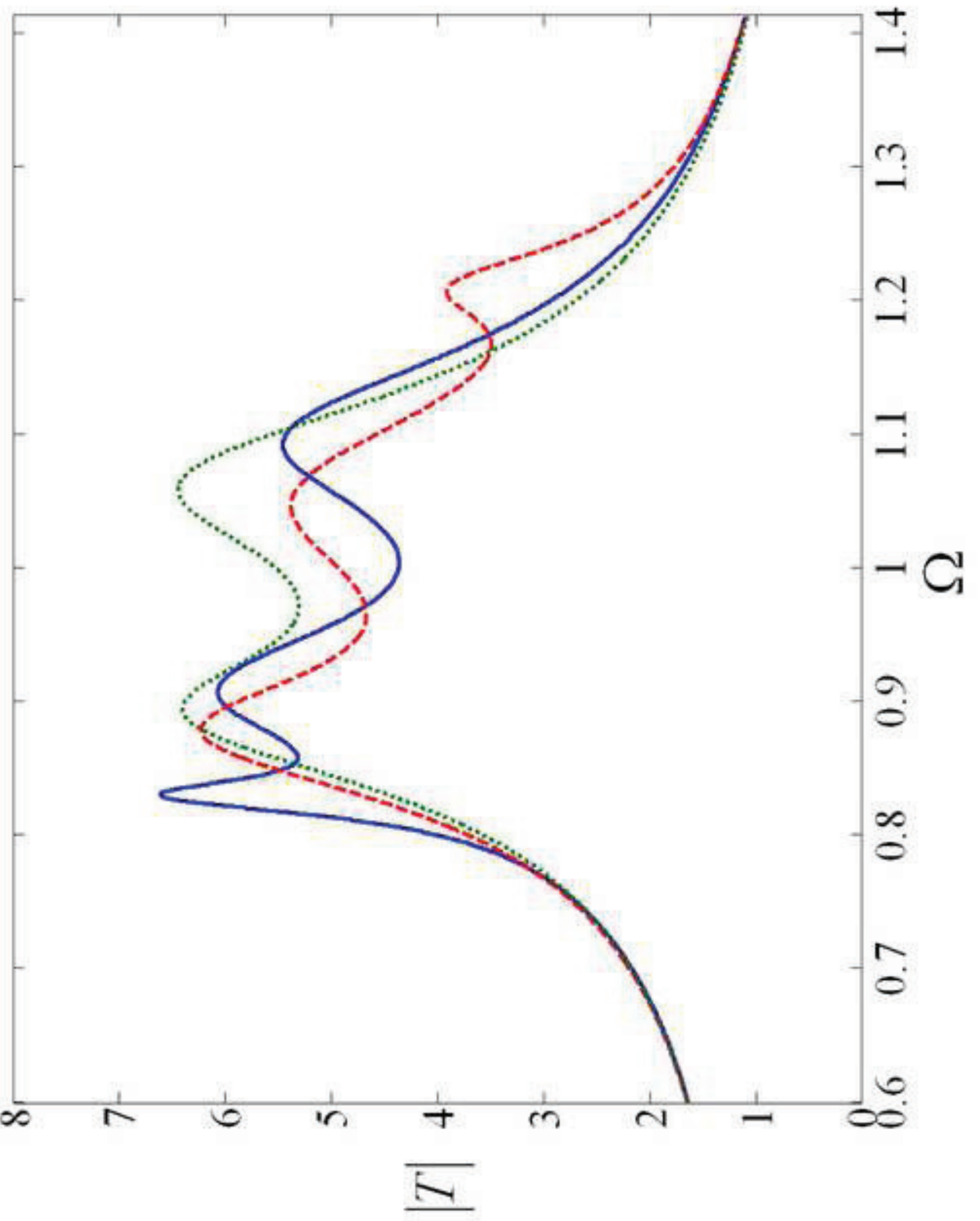

언 
Tables

The present method

Optimal $\|H\|_{2}[21]$

\begin{tabular}{lcc}
\hline$\mu$ & 0.05 & 0.05 \\
$\mu_{a}$ & 0.5964 & 0.3781 \\
$\gamma_{1}$ & 1.088 & 0.8528 \\
$v$ & 0.9220 & 1.0828 \\
$\zeta$ & 0.1510 & 0.1482
\end{tabular}

Table 1. Design parameters for a damped 2DOF TVA applied to a main structure 